\title{
German Heart Surgery Report 2016: The Annual Updated Registry of the German Society for Thoracic and Cardiovascular Surgery
}

\author{
Andreas Beckmann ${ }^{1}$ Anne-Katrin Funkat ${ }^{2}$ Jana Lewandowski ${ }^{1}$ Michael Frie ${ }^{3}$ Markus Ernst ${ }^{4}$ \\ Khosro Hekmat ${ }^{5}$ Wolfgang Schiller ${ }^{6}$ Jan F. Gummert ${ }^{7}$ Wolfgang Harringer ${ }^{8}$
}

${ }^{1}$ German Society for Thoracic and Cardiovascular Surgery, Langenbeck-Virchow-Haus, Berlin, Germany

2 Leipzig Heart Institute $\mathrm{GmbH}$, Leipzig, Germany

${ }^{3}$ FOM Hochschule für Oekonomie and Management, Essen, Germany

${ }^{4}$ Clinic for Cardiac and Vascular Surgery, University of Kiel,

Kiel, Germany

${ }^{5}$ Department of Cardiac and Thoracic Surgery, University of Cologne, Cologne, Germany

${ }^{6}$ Clinic for Cardiac Surgery, University of Bonn, Bonn, Germany

${ }^{7}$ Clinic for Thoracic and Cardiovascular Surgery, Heart and Diabetes Center NRW, Bad Oeynhausen, Germany

${ }^{8}$ Clinic for Cardiac, Thoracic and Vascular Surgery, Klinikum

Braunschweig gGmbH, Braunschweig, Germany

\begin{abstract}
Address for correspondence Andreas Beckmann, MD, Deutsche Gesellschaft für Thorax-, Herz- und Gefäßchirurgie [DGTHG], Langenbeck-Virchow-Haus, Luisenstr. 58-59, 10117 Berlin, Germany
\end{abstract} (e-mail: gf@dgthg.de).

Thorac Cardiovasc Surg 2017;65:505-518.

received

August 14, 2017

accepted after revision

August 29, 2017

published online

September 13, 2017
Based on a long-standing voluntary registry founded by the German Society for Thoracic and Cardiovascular Surgery (GSTCVS), well-defined data of all cardiac, thoracic, and vascular surgery procedures performed in 78 German heart surgery departments during the year 2016 are analyzed. In 2016, a total of 103,128 heart surgery procedures (implantable defibrillator, pacemaker, and extracardiac procedures excluded) were submitted to the registry. Approximately $15.7 \%$ of the patients were at least 80 years of age, resulting in an increase of $0.9 \%$ compared with the data of 2015 . For 37,614 isolated coronary artery bypass grafting procedures (relationship on-/offpump 4.4:1), an unadjusted in-hospital mortality of $2.9 \%$ was observed. Concerning the 33,451 isolated heart valve procedures (including 11,701 catheter-based procedures), the unadjusted in-hospital mortality was $4.3 \%$.

This annual updated registry of the GSTCVS represents voluntary public reporting by accumulating actual information for nearly all heart surgical procedures in Germany, describes advancements in heart medicine, and is a basis for internal and external quality assurances for all participants. In addition, the registry demonstrates that the provision of cardiac surgery in Germany is appropriate and patients are treated nationwide at all times. 


\section{Introduction}

Legitimate demands for a sophisticated quality management in medicine-by authorities, scientific organizations, health care companies, and patients all over the world-have stimulated a quality awareness resulting in the development of versatile quality assurance activities such as benchmark projects, registries, and others to answer those needs. As early as 1978, the board of directors of the German Society for Thoracic and Cardiovascular Surgery (GSTCVS, www. dgthg.de) decided to set up an annually updated data collection of all cardiac surgical procedures in terms of a voluntary registry. Since 1989 , the data are annually updated, summarized in registry format, and published in the scientific journal of the GSTCVS. ${ }^{1-27}$ The aims of this registry are to gather developments and upcoming trends in cardiac surgery in Germany; to compile various results for nearly all cardiac surgical procedures; to enable each participant a comparison of the reached individual institutional results to the nationwide achievements; and to facilitate an evaluation on an international level for the German society.

For monitoring actual conditions as well as developments in cardiac medicine, the registry covers all relevant techniques and also innovative technologies including minimally invasive cardiac surgery and all kinds of heart valve operations including transcatheter heart valve interventions (e.g. TAVI). Thereby, important findings for current patient safety and the future of patient care are collected and can be evaluated under different aspects.

Data and results presented in this report comprehend assorted data of the year 2016 .

\section{Materials and Methods}

Since 2004, a standardized questionnaire gathers specific information for well-defined procedures exactly described by an annually updated German adaption of the International Classification of Procedures in Medicine called operation code (Operationen- und Prozedurenschlüssel).

All participating institutions were requested to complete the structured questionnaire by January 22, 2017, entering all performed procedures and associated in-hospital mortality. The recommended path for data export is an electronic transmission of an encrypted file to the office of the GSTCVS in Berlin. After transaction, the data are decrypted, evaluated for completeness, and compiled for further analysis, thus ensuring anonymity for each participating institution. This compilation algorithm guarantees a high compliance for submission of complete datasets.

Inclusion criteria for the registry 2016 were all cardiac surgical procedures performed on patients between January 1, 2016, and December 31, 2016, unrelated to the date of admission or discharge of the patients as compared with other registries. Like in the earlier years, the number of procedures was counted rather than individual patients. For example, if during one admission a patient initially required isolated mitral valve reconstruction later followed by a coronary artery bypass grafting (CABG) due to an undesirable event, one count in the category "mitral valve reconstruction" and a second one in the category "coronary surgery" are enumerated. Thus, the registry contains more procedures than the real number of patients operated on.

Death of patients was defined as in-hospital mortality. Per definition, the observed mortality is always attributed to the first cardiac procedure, e.g. patient mortality will be attributed to an initial CABG procedure even if in a second procedure a replacement of the ascending aorta is performed due to a complication of the first operation. In case of fixing an undesirable event during the initial procedure, mortality will be counted under the more complex part of the operation.

The main reason for this structural setup of the registry -established over several decades-is to keep in accordance with the German data privacy act with its specific regulations for patients. Furthermore, it seemed to be relevant to get detailed information about all performed procedures and not only the number of treated patients. Last but not least, the process of data acquisition had to be standardized and feasible for all participants in Germany, thus enabling the submission of a complete dataset, regardless of the locally existing hardware and software used for data acquisition.

In 2016, a total of 78 institutions performed heart surgery. Fortunately, all departments answered the questionnaire and delivered a complete dataset for the year 2016 including hospital mortality rates.

\section{Registry Data 2016}

- Table 1 gives an overview of cardiac surgical procedures by distribution between the 16 German states, based on the population count of the Federal Office for Statistics as of December 31, 2014. In this table, the range of heart operations per 100,000 inhabitants shows a minimum of 105.9 (Baden Württemberg) and a maximum of 168.8 (Sachsen-Anhalt), resulting in a nationwide mean value of 125.7 (2015: 126.6). Analyzing the heart operations by department dimension, 56\% performed between 519 and 1,363 procedures (-Table 2 ). Summarizing the departments by various heart surgical procedures, it can be determined that heart operations in patients for congenital heart disease $(<1$ year, with extracorporeal circulation [ECC]) are conducted in 21 institutions and heart transplantations in 22 institutions (-Table 3 ), in near accordance to the previous year. - Table 4 illustrates the number of procedures using ECC from 2007 to 2016 in Germany. Over the past decade, the number of heart operations using ECC shows a diminution by 12,536 procedures, certainly an achievement of established innovations and minimally invasive therapies in heart surgery.

Overall, 184,789 procedures were reported to the registry for the year 2016 (-Table 5), a decrease of $0.3 \%$ (2015: 185,270 procedures). A total of 103,128 heart 
surgical procedures (excluded: implantable cardioverter defibrillator, pacemakers, and miscellaneous procedures without ECC) in 2016 display a comparable quantity with a difference of $0.8 \%(n=839)$ compared with 2015 (103,967 procedures) - Tables $\mathbf{6 ,} \mathbf{7}, \mathbf{V 1}$ to $\mathbf{~ 7 7 , ~ C 1 , ~ C 2 , ~}$ Con1, Con2, and Mis1 to Mis5 and - Figs. 1 to $\mathbf{9}$ demonstrate various compiled registry data concerning 2016 under different aspects and for various categories.

Compared with the data of previous years, several important developments continued in 2016 almost unchanged. The age distribution of patients ( - Fig. 6) shows a continuous shift to an elderly patient population with presently $52.8 \%$ of the cardiac procedures performed in patients of at least 70 years of age and $15.7 \%$ in octogenarians. However, mortality remained on the same low level over the represented decade (-Fig. 2). In this context it has to be noted that all designated mortality rates of this registry are unadjusted values. This must be taken into account for any interpretation. The rate of CABG procedures decreased over the past decade, while the relative number for off-pump CABG now reached a level of 18.5\% (2015: 16.5\%) (-Fig. 3).

A still ongoing trend is the increase of TAVI procedures in Germany (-Fig. 5, - Table V7), while the count of isolated aortic valve replacement procedures remained on a comparable level with just a slight decrease of 2.0\%. Starting in 2006 with just 78 implantations $(0.67 \%$ of isolated aortic valve procedures), in 2016 a total of 10,879 (49.8\%) TAVIs was reported to the registry. It must be emphasized that the 78 institutions which contribute their data to this registry do not represent all departments performing TAVI in Germany. In each case, the proceeding must correlate to the recommendations of international guidelines. ${ }^{28-30}$ For the German health service, a quality assurance directive for "minimally invasive heart valve interventions (TAVI, mitral valve clip reconstruction)" came into effect by law in July 2015, in which obligatory structures, defined processes, and qualified personnel are precisely specified. In addition to this registry responding to the directive of the Federal Joint Committee (G-BA), the results of the voluntary German Aortic Valve Registry $^{31-38}$ as well as the annual analyses for selected procedures in the context of the legally compulsory quality assurance ( $\$ 137$ SGB V) contribute to an exceptional patient benefit.

Since 2004 , more than $50 \%$ of isolated mitral valve procedures have been reconstructions. In 2016, a mitral valve reconstruction could be achieved in $62.9 \%$ of the procedures ( - Fig. 8). Based on the fact that all isolated mitral valve procedures are included without exception, regardless of the underlying valve disease concerning morphology or urgency of operation, it has to be assumed that the relative rate of mitral valve reconstruction would certainly be even higher if patients without possibility or indication for reconstruction would have been excluded (e.g., mitral valve stenosis, calcifications, or endocarditis). In other publications, for example, Gammie et al, ${ }^{39}$ patients with mitral valve stenosis, endocarditis, and emergency procedures are usually excluded. Therefore, the published rates of mitral valve repair have to be interpreted with caution if compared with this registry.

The continued increase of left ventricular assist device implantations (-Fig. 10) emphasizes the importance of mechanical circulatory support therapies, while biventricular assist device and total artificial heart implantations are of subordinate importance with only $3.6 \%$.

\section{Discussion}

The registry of the GSTCVS enables a comprehensive overview of all heart surgical procedures performed in Germany in 2016. The accuracy of this registry is considered to be high due to the implemented compilation algorithm using standardized operation coding which is a relevant criterion for reimbursement purposes. This is supported by other authors who could demonstrate a high accuracy for major outcome parameters in unaudited registries. ${ }^{40}$ In continuation with previous years, it can be concluded that heart surgery in Germany is performed on a constantly high level with superior in-hospital patient survival rates compared with international registries. In addition, the registry demonstrates that the German heart surgical provision is appropriate and the treatment of patients is guaranteed nationwide at any time (24/7/365). These aspects are especially important in the context of various activities in health care policy and the demographic change of the German population leading to an increase of patient age and related comorbidities and resulting in higher preoperative risk profile.

Compared with 2015, the number of cardiac surgery procedures remains on nearly the same level, due to the continuous increase of catheter-based heart valve procedures.

Further improvements in the basic configuration of the registry are recommended to enable more detailed and particularly risk-adjusted data analyses. However, if significant structural changes of data collection for the registry are conducted, it must be ensured that data compatibility still allows further longitudinal data analysis.

Completeness, validity, and further developments will depend on continued efforts of the GSTCVS in close collaboration with all cardiac surgical departments in Germany. This will be of outstanding importance in the sense of a contribution for patient safety and to obtain evidence for the high quality of heart surgery in Germany.

\section{Acknowledgments}

On behalf of the German Society for Thoracic and Cardiovascular Surgery, the authors would like to thank the heads of the departments and their employees of all cardiac surgery units in Germany for their continued cooperation and support to realize this registry. 


\section{Tables and Figures}

Table 1 Heart operations/German states

\begin{tabular}{|l|l|l|l|}
\hline German states & Quantity & Population & $\begin{array}{l}\text { Heart procedures/ } \\
\mathbf{1 0 0}\end{array}$ \\
\hline Baden Württemberg & & inhabitants \\
\hline Bayern & 11,348 & $10,716,644$ & 105.9 \\
\hline Berlin & 14,159 & $12,691,568$ & 111.6 \\
\hline Brandenburg & 3,897 & $3,469,849$ & 112.3 \\
\hline Bremen & 3,332 & $2,457,872$ & 135.6 \\
\hline Hamburg & 875 & 661,888 & 132.2 \\
\hline Hessen & 2,387 & $1,762,791$ & 135.4 \\
\hline Mecklenburg-Vorpommern & 7,599 & $6,093,888$ & 124.7 \\
\hline Niedersachsen & 1,926 & $1,599,138$ & 120.4 \\
\hline Nordrhein-Westfalen & 10,734 & $7,826,739$ & 137.1 \\
\hline Rheinland-Pfalz & 23,134 & $17,638,098$ & 131.2 \\
\hline Saarland & 5,360 & $4,011,582$ & 133.6 \\
\hline Sachsen & 1,471 & 989,035 & 148.7 \\
\hline Sachsen-Anhalt & 5,070 & $4,055,274$ & 125.0 \\
\hline Schleswig-Holstein & 3,773 & $2,235,548$ & 168.8 \\
\hline Thüringen & 4,078 & $2,830,864$ & 144.1 \\
\hline Deutschland & 2,945 & $2,156,759$ & 136.5 \\
\hline
\end{tabular}

${ }^{\mathrm{a}} 1,018$ foreign residents excluded.

${ }^{b}$ Federal Office for Statistics of Germany: Population; due date

December 31, 2014.

Table 2 Units assorted by quantified categories $\left(\sum^{\mathrm{a}}[n=103,128]\right)$

\begin{tabular}{|l|l|l|l|l|l|}
\hline $\begin{array}{l}\text { Procedures } \\
\text { (quantity) }\end{array}$ & $<500$ & $\mathbf{5 0 0 - 9 9 9}$ & $\mathbf{1 , 0 0 0 - 1 , 4 9 9}$ & $\mathbf{1 , 5 0 0 - 1 , 9 9 9}$ & $\mathbf{2 , 0 0 0 - 5 , 0 0 0}$ \\
\hline $\begin{array}{l}\text { Departments } \\
\text { (number) }\end{array}$ & 8 & 21 & 23 & 13 & 13 \\
\hline Average & 387 & 817 & 1,156 & 1,698 & 2,632 \\
\hline Range & $216-490$ & $519-996$ & $1,019-1,363$ & $1,517-1,926$ & $2,008-3,939$ \\
\hline
\end{tabular}

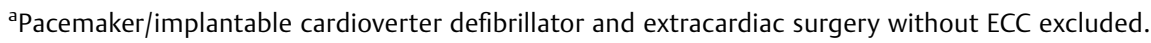

Table 3 Departments summarized by heart surgery procedures 2016

\begin{tabular}{|l|l|}
\hline Type of surgery performed & $N$ \\
\hline Coronary artery bypass grafting & 77 \\
\hline Heart valve surgery & 77 \\
\hline Surgery for CHD in pts $<1$ y with ECC & $21^{\text {a }}$ \\
\hline HTx & $22^{\text {b }}$ \\
\hline HLTx & 4 \\
\hline
\end{tabular}

Abbreviations: CHD, congenital heart disease; ECC, extracorporeal circulation; HTx, heart transplantation; HLTx, heart-lung transplantation; pts, patients; Tx, transplantation.

${ }^{\mathrm{a}} \mathrm{N}=2,130$ : thereof: 15 to 19 procedures in 3 units, 30 to 49 procedures in 4 units, 57 to 94 procedures in 4 units, and 100 to 274 procedures in 10 units.

${ }^{b} N=291$ : thereof: 1 to 3 Tx in four units, 5 to 9 Tx in eight units, 10 to $19 \mathrm{Tx}$ in eight units, and 23 to 82 Tx in two units. 
Table 4 Cardiac procedures using extracorporeal circulation (2007-2016)

\begin{tabular}{|l|l|l|l|l|l|l|l|l|l|l|}
\hline & 2007 & 2008 & 2009 & 2010 & 2011 & 2012 & 2013 & 2014 & 2015 & 2016 \\
\hline Units & 80 & 79 & 80 & 79 & 78 & 79 & 79 & 78 & 78 & 78 \\
\hline Operations & 91,618 & 89,773 & 86,916 & 84,686 & 84,402 & 84,388 & 84,040 & 83,787 & 81,527 & 79,082 \\
\hline Average & 1,145 & 1,136 & 1,086 & 1,072 & 1,082 & 1,068 & 1,064 & 1,074 & 1,045 & 1,014 \\
\hline
\end{tabular}

Table 5 Frequency of cardiac procedures 2016

\begin{tabular}{|l|l|l|l|l|}
\hline Category & $\begin{array}{l}\text { With } \\
\text { ECC }\end{array}$ & $\begin{array}{l}\text { Without } \\
\text { ECC }\end{array}$ & Total & $\begin{array}{l}\text { Difference } \\
\mathbf{2 0 1 5}(\%)\end{array}$ \\
\hline Coronary artery bypass grafting & $42,813^{*}$ & $7,301^{*}$ & 50,114 & -3.5 \\
\hline Heart valve procedures & $21,877^{*}$ & $11,574^{*}$ & 33,451 & +3.4 \\
\hline Surgery of thoracic aorta & $7,369^{*}$ & $750^{*}$ & 8,119 & +2.5 \\
\hline Surgery for CHD & $4,762^{*}$ & $1,093^{*}$ & 5,855 & -0.6 \\
\hline Cardiac surgery, other & $1,072^{*}$ & $1,183^{*}$ & 2,255 & -5.2 \\
\hline Assist device procedures** & $789^{*}$ & $2,145^{*}$ & 2,934 & -4.6 \\
\hline Extracardiac surgery & $358^{*}$ & 57,023 & 57,381 & +4.1 \\
\hline Pacemaker and ICD procedures & $42^{*}$ & 24,638 & 24,680 & -7.1 \\
\hline Total & 79,082 & 105,707 & 184,789 & -0.3 \\
\hline
\end{tabular}

*Sum: $\mathbf{n}=103,128$ (heart surgery procedures).

**Sum: VADs / TAH / others devises (e.g. ECLS/ECMO).

Abbreviations: CHD, congenital heart disease; ECC, extracorporeal circulation; ICD, implantable cardioverter defibrillator; VADs; Ventricular assist devices; TAH, total artificial heart; ECLS, Extracoporeal life support; ECMO, Extracorporeal membrane oxygenation.

Table 6 Additional data for heart procedures with ECC in 2016 versus 2015

\begin{tabular}{|l|l|l|l|l|}
\hline Procedures & \multicolumn{2}{|l|}{2016} & 2015 & \multicolumn{1}{l|}{} \\
\hline Emergency & 12,745 & $12.4 \%$ & 12,843 & $12.4 \%$ \\
\hline Redo & 9,186 & $8.9 \%$ & 8,994 & $8.7 \%$ \\
\hline
\end{tabular}

Abbreviation: ECC, extracorporeal circulation.

Table 7 Gender distribution

\begin{tabular}{|l|l|l|}
\hline & Female (\%) & Male (\%) \\
\hline Heart valve procedures & 43 & 57 \\
\hline Coronary procedures & 23 & 77 \\
\hline Surgery for CHD & 45 & 55 \\
\hline Surgery of thoracic aorta & 34 & 66 \\
\hline Cardiac surgery, other & 57 & 43 \\
\hline Assist devices & 26 & 74 \\
\hline Pacemaker and ICD & 36 & 64 \\
\hline Extracardiac surgery & 35 & 65 \\
\hline Total & 34 & 66 \\
\hline
\end{tabular}

Abbreviations: CHD, congenital heart disease; ICD, implantable cardioverter defibrillator.
Table V1 Isolated valve procedures

\begin{tabular}{|l|l|l|l|}
\hline Procedures & $N$ & $\dagger$ & $\%$ \\
\hline Single & 17,855 & 680 & 3.8 \\
\hline Double & 3,298 & 323 & 9.8 \\
\hline Triple & 409 & 59 & 14.4 \\
\hline $\begin{array}{l}\text { Transcatheter access } \\
\text { (single valve) }\end{array}$ & 11,701 & 363 & 3.1 \\
\hline $\begin{array}{l}\text { Transcatheter access } \\
\text { (double valve) }\end{array}$ & 15 & 2 & 13.3 \\
\hline Not specified & 173 & 9 & 5.2 \\
\hline Total & 33,451 & 1,436 & 4.3 \\
\hline
\end{tabular}

Note: Transcatheter valve procedures: 10,879 aortic valve implantations; 147 mitral valve implantations; 650 mitral valve repairs; 2 tricuspid valve implantations; 23 tricuspid valve repairs; 15 double aortic and mitral valve procedures; no pulmonary valve implantation. 
Table V2 Single heart valve procedures: access type

\begin{tabular}{|l|l|l|l|}
\hline Heart valve/access & $N$ & $\dagger$ & $\%$ \\
\hline Aortic valve & 21,963 & 680 & 3.1 \\
\hline Sternotomy & 7,879 & 310 & 3.9 \\
\hline Partial sternotomy & 3,205 & 45 & 1.4 \\
\hline Transvascular & 8,792 & 231 & 2.6 \\
\hline Transapical & 2,087 & 94 & 4.5 \\
\hline Mitral valve & 7,014 & 303 & 4.3 \\
\hline Sternotomy & 3,115 & 214 & 6.9 \\
\hline Minimal invasive & 3,102 & 52 & 1.7 \\
\hline Transcatheter & 797 & 37 & 4.6 \\
\hline Tricuspid valve & 531 & 58 & 10.9 \\
\hline Sternotomy & 381 & 47 & 12.3 \\
\hline Minimal invasive & 125 & 10 & 8.0 \\
\hline Transcatheter & 25 & 1 & 4.0 \\
\hline Pulmonary valve & 48 & 2 & 4.2 \\
\hline Sternotomy & 48 & 2 & 4.2 \\
\hline Minimal invasive & 0 & 0 & - \\
\hline Transcatheter & 0 & 0 & - \\
\hline Total & 29,556 & 1,043 & 3.5 \\
\hline
\end{tabular}

Table V3 Isolated aortic valve procedures

\begin{tabular}{|l|l|l|l|}
\hline Prosthesis/native valve & $N$ & $\dagger$ & $\%$ \\
\hline Xenograft & 9,722 & 322 & 3.3 \\
\hline Mechanical prosthesis & 1,201 & 28 & 2.3 \\
\hline Repair & 123 & 2 & 1.6 \\
\hline Homograft & 38 & 3 & 7.9 \\
\hline Total & 11,084 & 355 & 3.2 \\
\hline
\end{tabular}

Table V4 Isolated mitral valve procedures

\begin{tabular}{|l|l|l|l|}
\hline Prosthesis/native valve & $N$ & $\dagger$ & $\%$ \\
\hline Repair & 3,908 & 49 & 1.3 \\
\hline Xenograft & 1,821 & 190 & 10.4 \\
\hline Mechanical prosthesis & 473 & 26 & 5.5 \\
\hline Homograft & 15 & 1 & 6.7 \\
\hline Total & 6,217 & 266 & 4.3 \\
\hline
\end{tabular}

Note: Transcatheter procedures are excluded.

Table V5 Multiple heart valve procedures

\begin{tabular}{|l|l|l|l|}
\hline Combination & $N$ & $\dagger$ & $\%$ \\
\hline Mitral + tricuspid & 1,665 & 142 & 8.5 \\
\hline Aortic + mitral & 1,401 & 157 & 11.2 \\
\hline Aortic + mitral + tricuspid & 407 & 58 & 14.3 \\
\hline Aortic + tricuspid & 160 & 24 & 15.0 \\
\hline Aortic + pulmonary ${ }^{\text {a }}$ & 54 & 0 & 0.0 \\
\hline Tricuspid + pulmonary & 18 & 0 & 0.0 \\
\hline Aortic + mitral + pulmonary & 2 & 1 & 50.0 \\
\hline Total & 3,707 & 382 & 10.3 \\
\hline
\end{tabular}

Note: Transcatheter procedures excluded.

ancluding Ross procedures.

Table V6 Isolated/combined mitral valve surgery-implantation/replacement versus repair

\begin{tabular}{|c|l|l|l|l|l|l|l|l|l|l|}
\hline \multirow{2}{*}{ Mitral valve surgery } & \multicolumn{3}{l|}{ Repair } & \multicolumn{3}{l|}{$\begin{array}{l}\text { Implantation/ } \\
\text { replacement }\end{array}$} \\
\cline { 2 - 12 } & $N$ & $\dagger$ & $\%$ & $N$ & $\dagger$ & $\%$ & $N$ & $\%$ repair & $\dagger$ & $\%$ \\
\hline Isolated & 3,908 & 49 & 1.3 & 2,309 & 217 & 9.4 & 6,217 & 62,9 & 266 & 4.3 \\
\hline+ Aortic valve & 563 & 42 & 7.5 & 838 & 115 & 13.7 & 1,401 & 40,2 & 157 & 11.2 \\
\hline+ Tricuspid valve repair ${ }^{a}$ & 977 & 44 & 4.5 & 631 & 86 & 13.6 & 1,608 & 60,8 & 130 & 8.1 \\
\hline+ CABG & 1,585 & 107 & 6.8 & 976 & 157 & 16.1 & 2,561 & 61,9 & 264 & 10.3 \\
\hline+ CABG + aortic valve replacement & 310 & 30 & 9.7 & 309 & 64 & 20.7 & 619 & 50,1 & 94 & 15.2 \\
\hline Total & 7,343 & 272 & 3.7 & 5,063 & 639 & 12.6 & 12,406 & 59,2 & 911 & 7.3 \\
\hline
\end{tabular}

a 57 procedures (not specified mitral valve + tricuspid valve surgery) were excluded; $\uparrow: 21 \%(12 / 57)$. 
Table V7 Transcatheter heart valve procedures

\begin{tabular}{|c|c|c|c|c|c|c|c|}
\hline & \multicolumn{2}{|c|}{ Without ECC } & \multicolumn{2}{|c|}{ With ECC } & \multicolumn{3}{|c|}{ All procedures } \\
\hline & $N$ & $\dagger$ & $N$ & $\dagger$ & $N$ & $\dagger$ & $\%$ \\
\hline Aortic valve implantation & 10,765 & 290 & 114 & 35 & 10,879 & 325 & 3 \\
\hline Transvascular & 8,731 & 206 & 61 & 25 & 8,792 & 231 & 3 \\
\hline Transapical & 2,034 & 84 & 53 & 10 & 2,087 & 94 & 5 \\
\hline Mitral valve & 770 & 31 & 27 & 6 & 797 & 37 & 5 \\
\hline Repair & 628 & 20 & 22 & 1 & 650 & 21 & 3 \\
\hline Implantation & 142 & 11 & 5 & 5 & 147 & 16 & 11 \\
\hline Tricuspid valve repair & 24 & 1 & 1 & 0 & 25 & 1 & 4 \\
\hline Repair & 22 & 1 & 1 & 0 & 23 & 1 & 4 \\
\hline Implantation & 2 & 0 & 0 & 0 & 2 & 0 & 0 \\
\hline Aortic + mitral valve implantation & 15 & 2 & 0 & 0 & 15 & 2 & 13 \\
\hline Aortic valve implantation $^{\mathrm{a}}+\mathrm{CABC}$ & 33 & 4 & 16 & 6 & 49 & 10 & 20 \\
\hline Mitral valve implantation ${ }^{\mathrm{b}}+\mathrm{CABG}$ & 0 & 0 & 3 & 1 & 3 & 1 & 33 \\
\hline Aortic + mitral valve + CABG & 0 & 0 & 0 & 0 & 0 & 0 & - \\
\hline Total & 11,607 & 328 & 161 & 48 & 11,768 & 376 & 3 \\
\hline
\end{tabular}

Abbreviations: CABG, coronary artery bypass grafting; ECC, extracorporeal circulation.

Notes: Pulmonary valve implantation for the correction of congenital lesions is not included, No procedure was reported for adults without congenital lesion, $19 \%$ of transcatheter aortic valve implantation (TAVI) by transapical access; and 1\% of TAVI under use of ECC.

${ }^{\mathrm{a}}$ Femoral, subclavian, or transaortic access.

${ }^{\mathrm{b}}$ Transvascular and transapical access.

Table C1 Isolated CABG and combined procedures with ECC

\begin{tabular}{|l|l|l|l|}
\hline Procedures & N & $\dagger$ & $\%$ \\
\hline Isolated CABG & 37,614 & 1,080 & 2.9 \\
\hline+ aortic valve replacement & 6,742 & 321 & 4.8 \\
\hline+ other & 2,401 & 154 & 6.4 \\
\hline+ mitral valve repair & 1,585 & 107 & 6.8 \\
\hline+ mitral valve replacement & 976 & 157 & 16.1 \\
\hline+ aortic valve replacement + mitral valve repair & 310 & 30 & 9.7 \\
\hline+ aortic + mitral valve replacement & 309 & 64 & 20.7 \\
\hline+ aneurysm resection & 125 & 9 & 7.2 \\
\hline+ transcatheter aortic valve implantation & 49 & 10 & 20.4 \\
\hline Total & 50,111 & 1,932 & 3.9 \\
\hline
\end{tabular}

Abbreviations: $C A B G$, coronary artery bypass grafting; ECC, extracorporeal circulation.

Table C2 Isolated CABG with/without ECC

\begin{tabular}{|c|c|c|c|c|c|c|c|c|c|}
\hline \multirow[t]{2}{*}{ Grafts (n) } & \multicolumn{3}{|c|}{ With ECC } & \multicolumn{3}{|c|}{ Without ECC } & \multicolumn{3}{|l|}{ All } \\
\hline & $N$ & $\dagger$ & $\%$ & $N$ & $\dagger$ & $\%$ & $N$ & $\dagger$ & $\%$ \\
\hline Single & 1,013 & 59 & 5.8 & 1,488 & 45 & 3.0 & 2,501 & 104 & 4.2 \\
\hline Double & 6,604 & 247 & 3.7 & 1,941 & 36 & 1.9 & 8,545 & 283 & 3.3 \\
\hline Triple & 13,524 & 405 & 3.0 & 2,361 & 35 & 1.5 & 15,885 & 440 & 2.8 \\
\hline Quadruple & 7,261 & 178 & 2.5 & 961 & 15 & 1.6 & 8,222 & 193 & 2.3 \\
\hline Quintuple + more & 2,254 & 59 & 2.6 & 207 & 1 & 0.5 & 2,461 & 60 & 2.4 \\
\hline Total & 30,656 & 948 & 3.1 & 6,958 & 132 & 1.9 & 37,614 & 1,080 & 2.9 \\
\hline
\end{tabular}

Abbreviations: CABG, coronary artery bypass grafting; ECC, extracorporeal circulation. 
512 German Heart Surgery Report 2016 Beckmann et al.

Table Con1 Congenital heart surgery with/without ECC

\begin{tabular}{|l|l|l|l|l|l|l|}
\hline \multirow{2}{*}{ Age $(\mathrm{y})$} & \multicolumn{2}{|l|}{$\boldsymbol{\dagger}$} & $\dagger$ & $\%$ & \% \\
\cline { 2 - 7 } & With ECC & Without ECC & With ECC & Without ECC & With ECC & Without ECC \\
\hline$<1$ & 2,154 & 835 & 80 & 25 & 3.7 & 3.0 \\
\hline $1-17$ & 1,672 & 238 & 18 & 3 & 1.1 & 1.3 \\
\hline$\geq 18$ & 1,001 & 20 & 33 & 1 & 3.3 & 5.0 \\
\hline Total & 2,673 & 258 & 51 & 4 & 1.9 & 1.6 \\
\hline
\end{tabular}

Abbreviation: ECC, extracorporeal circulation.

Table Con2 Procedures for congenital heart disease with and without ECC

\begin{tabular}{|c|c|c|c|c|c|c|c|c|c|}
\hline \multirow[t]{2}{*}{ Lesion/procedure } & \multicolumn{3}{|c|}{ Age $<1$ y } & \multicolumn{3}{|c|}{ Age 1-17 y } & \multicolumn{3}{|c|}{ Age $\geq 18 y$} \\
\hline & $N$ & $\dagger$ & $\%$ & $N$ & $\dagger$ & $\%$ & $N$ & $\dagger$ & $\%$ \\
\hline ASD & 35 & 0 & 0.0 & 245 & 0 & 0.0 & 272 & 4 & 1.5 \\
\hline Complete AV canal & 213 & 5 & 2.3 & 75 & 1 & 1.3 & 13 & 0 & 0.0 \\
\hline VSD & 295 & 0 & 0.0 & 110 & 0 & 0.0 & 20 & 0 & 0.0 \\
\hline Fallot's tetralogy & 188 & 3 & 1.6 & 52 & 0 & 0.0 & 5 & 0 & 0.0 \\
\hline DORV & 56 & 2 & 3.6 & 15 & 0 & 0.0 & 1 & 0 & 0.0 \\
\hline TGA & 171 & 1 & 0.6 & 3 & 0 & 0.0 & 0 & 0 & - \\
\hline TGA + VSD & 54 & 3 & 5.6 & 5 & 0 & 0.0 & 0 & 0 & - \\
\hline Truncus arteriosus & 35 & 2 & 5.7 & 8 & 0 & 0.0 & 1 & 0 & 0.0 \\
\hline Fontan & 2 & 0 & 0.0 & 214 & 1 & 0.5 & 8 & 1 & 12.5 \\
\hline Norwood & 183 & 30 & 16.4 & 2 & 1 & 50.0 & 0 & 0 & - \\
\hline Pulmonary valve & 64 & 1 & 1.6 & 199 & 2 & 1.0 & 71 & 4 & 5.6 \\
\hline $\begin{array}{l}\text { Transcatheter pulmonary } \\
\text { valve implantation }\end{array}$ & 0 & - & - & 6 & 0 & 0.0 & 3 & 0 & 0.0 \\
\hline Aortic valve & 50 & 1 & 2.0 & 188 & 5 & 2.7 & 347 & 8 & 2.3 \\
\hline Ross procedure & 9 & 0 & 0.0 & 29 & 0 & 0.0 & 29 & 0 & 0.0 \\
\hline Mitral valve & 35 & 1 & 2.9 & 100 & 0 & 0.0 & 86 & 9 & 10.5 \\
\hline Tricuspid valve & 68 & 3 & 4.4 & 58 & 2 & 3.4 & 45 & 3 & 6.7 \\
\hline PDA & 214 & 8 & 3.7 & 28 & 0 & 0.0 & 1 & 0 & 0.0 \\
\hline Coarctation & 208 & 1 & 0.5 & 38 & 0 & 0.0 & 3 & 0 & 0.0 \\
\hline Others & 1,105 & 44 & 4.0 & 481 & 7 & 1.5 & 116 & 5 & 4.3 \\
\hline $\mathrm{HTx}$ & 4 & 0 & 0.0 & 38 & 1 & 2.6 & 0 & 0 & - \\
\hline HLTx & 0 & - & - & 2 & 0 & 0.0 & 0 & 0 & - \\
\hline LTx & 0 & - & - & 14 & 1 & 7.1 & 0 & 0 & - \\
\hline Total & 2,989 & 105 & 3.5 & 1,910 & 21 & 1.1 & 1,021 & 34 & 3.3 \\
\hline
\end{tabular}

Abbreviations: ASD, atrial septal defect; AV, atrioventricular; DORV, double outlet right ventricle; ECC, extracorporeal circulation; HLTx, heart-lung transplantation; HTx, heart transplantation; LTx, lung transplantation; PDA, patent ductus arteriosus; TGA, transposition of the great arteries; VSD, ventricular septal defect.

Table Mis1 Ross procedures (autologous aortic and pulmonary valve replacement)

\begin{tabular}{|l|l|l|l|l|l|l|l|l|l|l|}
\hline Age (y) & $\mathbf{2 0 0 7}$ & $\mathbf{2 0 0 8}$ & $\mathbf{2 0 0 9}$ & $\mathbf{2 0 1 0}$ & $\mathbf{2 0 1 1}$ & $\mathbf{2 0 1 2}$ & $\mathbf{2 0 1 3}$ & $\mathbf{2 0 1 4}$ & $\mathbf{2 0 1 5}$ & $\mathbf{2 0 1 6}$ \\
\hline$<18$ & 34 & 42 & 54 & 43 & 40 & 36 & 33 & 37 & 28 & 38 \\
\hline$\geq 18$ & 261 & 207 & 175 & 184 & 134 & 117 & 107 & 90 & 64 & 72 \\
\hline Total & 295 & 249 & 229 & 227 & 174 & 153 & 140 & 127 & 92 & 110 \\
\hline
\end{tabular}

Abbreviation: AV, aortic valve. 
Table Mis2 Heart and lung transplantation

\begin{tabular}{|c|c|c|c|c|c|c|}
\hline \multirow[t]{2}{*}{ Organ } & \multicolumn{3}{|c|}{ With ECC } & \multicolumn{3}{|c|}{ Without ECC } \\
\hline & $N$ & $\dagger$ & $\%$ & $N$ & $\dagger$ & $\%$ \\
\hline HTx & 291 & 38 & 13.1 & & & \\
\hline HLTX & 7 & 0 & 0.0 & & & \\
\hline LTx & 53 & 10 & 18.9 & 247 & 14 & 5.7 \\
\hline
\end{tabular}

Abbreviations: HTx, heart transplantation; HLTx, heart-lung transplantation; LTx, lung transplantation; Tx, transplantation.

Notes: All pediatric transplantations (demonstrated in - Table Con2) are included in this table, Eurotransplant report 2016: 287 HTx, 2

HTx + kidneyTx, 1 HTx + liverTx, 7 HLTx, 281 double lung transplantation, 38 single lung transplantation, 0 LTx + kidneyTx, and 2 LTx + liverTx.

Table Mis3 Aortic surgery

\begin{tabular}{|c|c|c|c|c|c|c|}
\hline \multirow[t]{2}{*}{ Replacement $^{\mathrm{a}}$} & \multicolumn{3}{|c|}{ With ECC } & \multicolumn{3}{|c|}{ Without ECC } \\
\hline & $N$ & $\dagger$ & $\%$ & $N$ & $\dagger$ & $\%$ \\
\hline Supracoronary replacement of ascending aorta & 1,383 & 128 & 9.3 & & & \\
\hline Supracoronary ascending + aortic valve replacement & 1,325 & 69 & 5.2 & & & \\
\hline \multicolumn{7}{|l|}{ Infracoronary ascending } \\
\hline Mechanical valve conduits & 441 & 33 & 7.5 & & & \\
\hline Biological valve conduits & 999 & 110 & 11.0 & & & \\
\hline David procedure & 537 & 15 & 2.8 & & & \\
\hline Yacoub procedure & 120 & 5 & 4.2 & & & \\
\hline Other & 286 & 21 & 7.3 & & & \\
\hline Aortic arch replacement ${ }^{\mathrm{b}}$ & 2,117 & 246 & 11.6 & & & \\
\hline Replacement of descending aorta & 43 & 20 & 46.5 & 7 & 1 & 14.3 \\
\hline Thoracoabdominal aortic replacement & 101 & 16 & 15.8 & 24 & 5 & 20.8 \\
\hline Endostent descending aorta & 17 & 0 & 0.0 & 719 & 46 & 6.4 \\
\hline Total & 7,369 & 663 & 9.0 & 750 & 52 & 6.9 \\
\hline
\end{tabular}

Abbreviation: ECC, extracorporeal circulation.

Notes: All procedures involving aortic surgery are included in this table, isolated aortic surgery as well as all possible combined procedures (e.g., additional coronary artery bypass grafting) are summarized in this category.

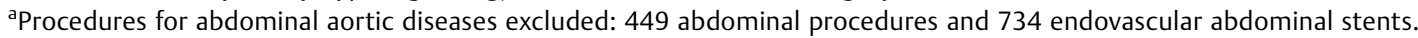

${ }^{\mathrm{b}}$ All possible combined procedures included, the only common denominator is aortic arch surgery.

Table Mis4 Pacemaker and ICD procedures

\begin{tabular}{|l|l|l|l|l|l|l|l|}
\hline \multirow{2}{*}{ Device/category } & \multicolumn{3}{|l|}{ With ECC } & \multicolumn{2}{l|}{ Without ECC } \\
\cline { 2 - 8 } & $N$ & $\dagger$ & $\%$ & $N$ & $\dagger$ & $N$ & $\dagger$ \\
\hline Pacemaker & 13,882 & 118 & 0.9 & 25 & 4 & 13,857 & 114 \\
\hline Implantation & 8,968 & 71 & 0.8 & 8 & 1 & 8,960 & 70 \\
\hline Battery exchange & 1,907 & 2 & 0.1 & 2 & 0 & 1,905 & 2 \\
\hline Revision procedures & 3,007 & 45 & 1.5 & 15 & 3 & 2,992 & 42 \\
\hline ICD & 9,368 & 85 & 0.9 & 17 & 2 & 9,351 & 83 \\
\hline Implantation & 4,140 & 21 & 0.5 & 2 & 1 & 4,138 & 20 \\
\hline Battery exchange & 2,019 & 3 & 0.1 & 0 & 0 & 2,019 & 3 \\
\hline Revision procedures & 3,209 & 61 & 1.9 & 15 & 1 & 3,194 & 60 \\
\hline Miscellaneous & 1,430 & 11 & 0.8 & 0 & 0 & 1,430 & 11 \\
\hline Total & 24,680 & 214 & 0.9 & 42 & 6 & 24,638 & 208 \\
\hline
\end{tabular}

Abbreviations: ECC, extracorporeal circulation; ICD, implantable cardioverter defibrillator. 
514 German Heart Surgery Report 2016 Beckmann et al.

Table Mis5 Surgical ablation procedures for tachyarrhythmia

\begin{tabular}{|l|l|l|l|}
\hline Energy source & Total & Endocardiac & Epicardiac \\
\cline { 3 - 4 } & & $N$ & $N$ \\
\hline Unipolar radiofrequency & 200 & 65 & 135 \\
\hline Unipolar cryo-radiofrequency & 241 & 109 & 132 \\
\hline Bipolar radiofrequency & 2,210 & 223 & 1,987 \\
\hline Cryothermy & 1,523 & 1,307 & 216 \\
\hline Microwave & 13 & 3 & 10 \\
\hline Focused ultrasound & 89 & 22 & 67 \\
\hline Laser & 0 & 0 & 0 \\
\hline Other & 29 & 14 & 15 \\
\hline Total & 4,305 & 1,743 & 2,562 \\
\hline
\end{tabular}

Note: 419 procedures are not specified with regard to endocardiac/epicardiac ablation.

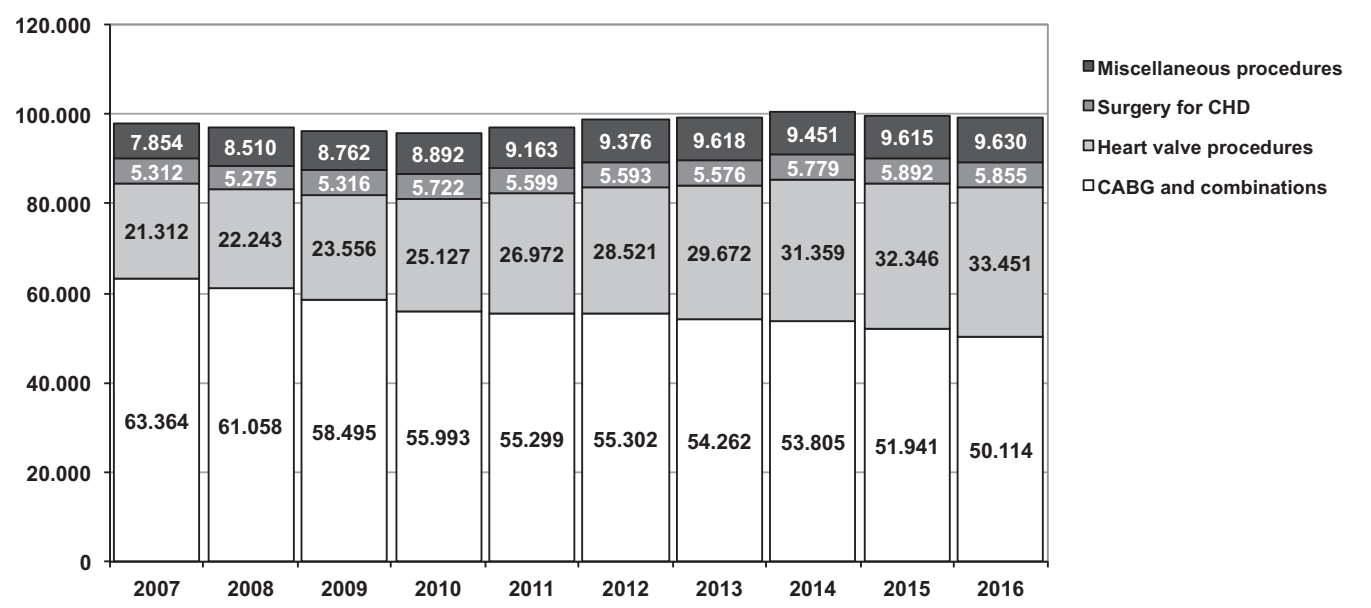

Fig. 1 Selected heart surgical categories (2007-2016). (1) CABG and combined procedures: all types of coronary surgery. (2) Heart valve procedures: combination with aortic surgery summarized under miscellaneous procedures. (3) Congenital heart surgery: ASD repairs in adults or in combination with $C A B G$ or heart valve procedures are summarized in the CABG or heart valve procedure groups. (4) Miscellaneous procedures: all other types of procedures with ECC. ASD, atrial septal defect; CABG, coronary artery bypass grafting; ECC, extracorporeal circulation.
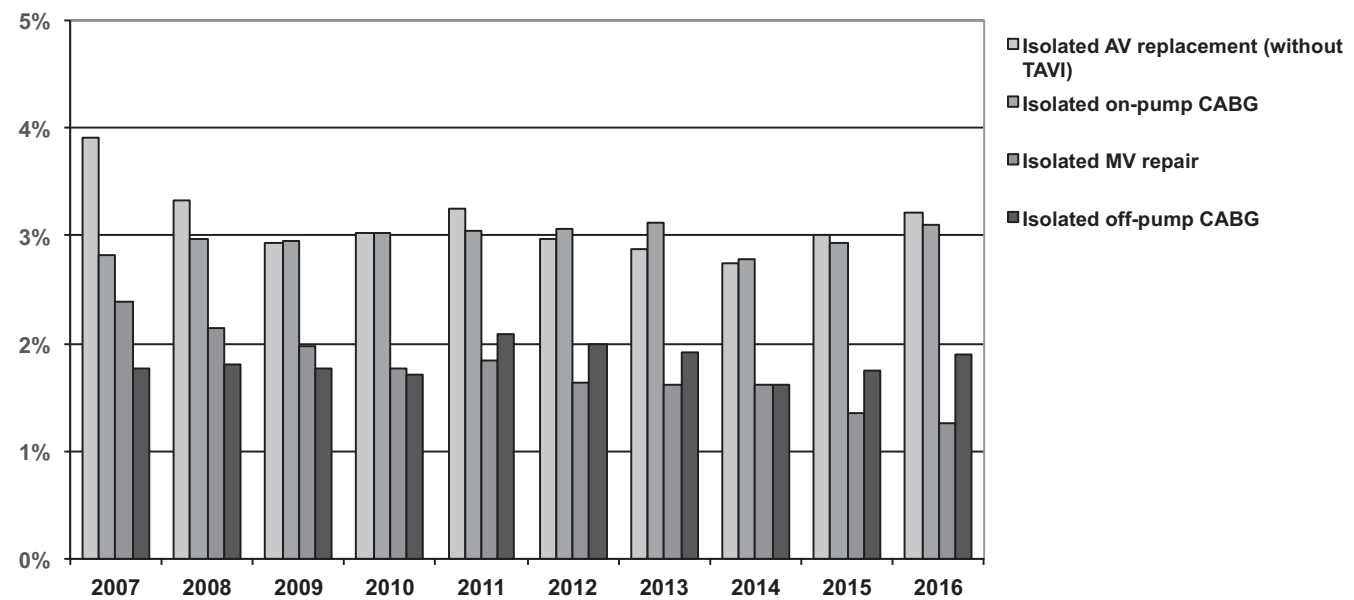

Fig. 2 Unadjusted mortality for selected procedures (2007-2016). AV, aortic valve; CABG, coronary artery bypass grafting; MV, mitral valve; TAVI, transcatheter heart valve intervention. 
German Heart Surgery Report 2016 Beckmann et al. 515

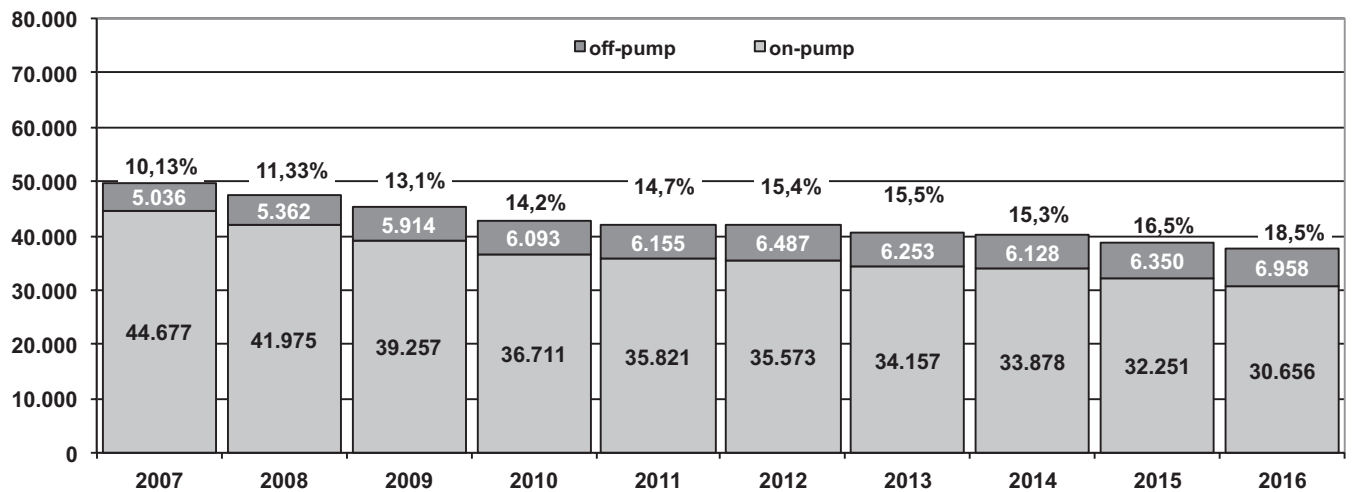

Fig. 3 Isolated coronary artery bypass grafting (2007-2016).

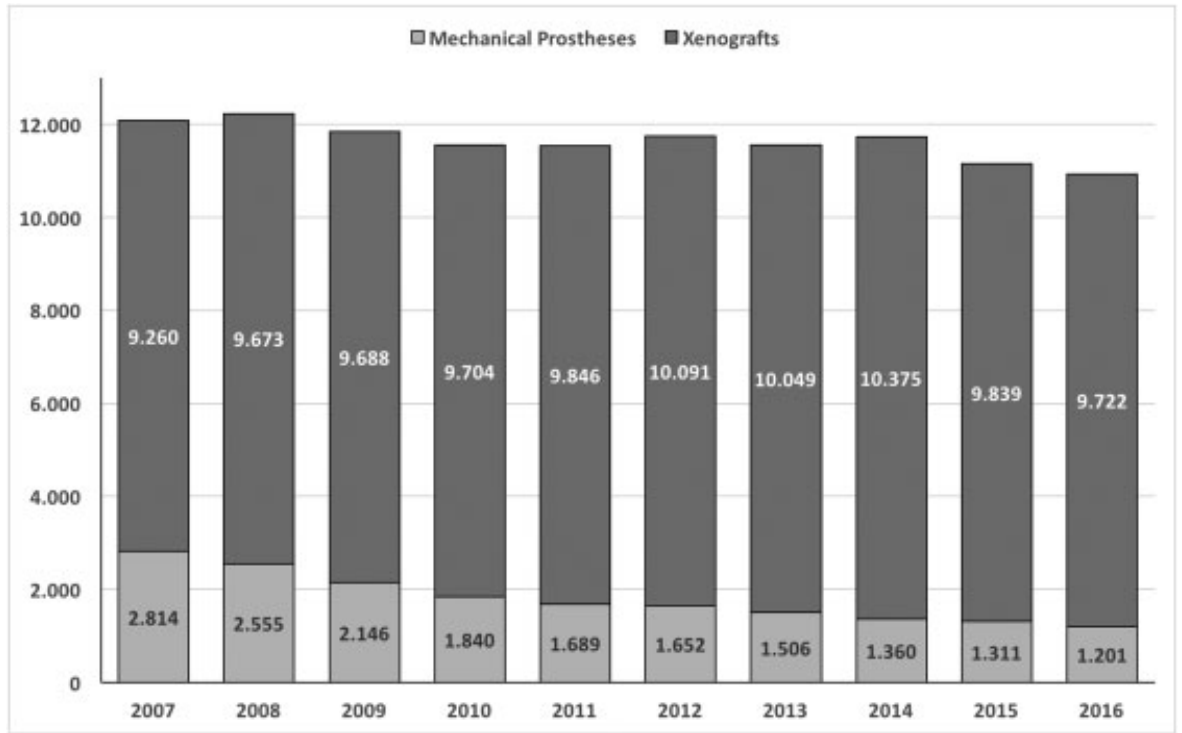

Fig. 4 Isolated aortic valve replacement (2007-2016). Notes: Ross procedures, homograft implantations, and transcatheter heart valve intervention excluded.

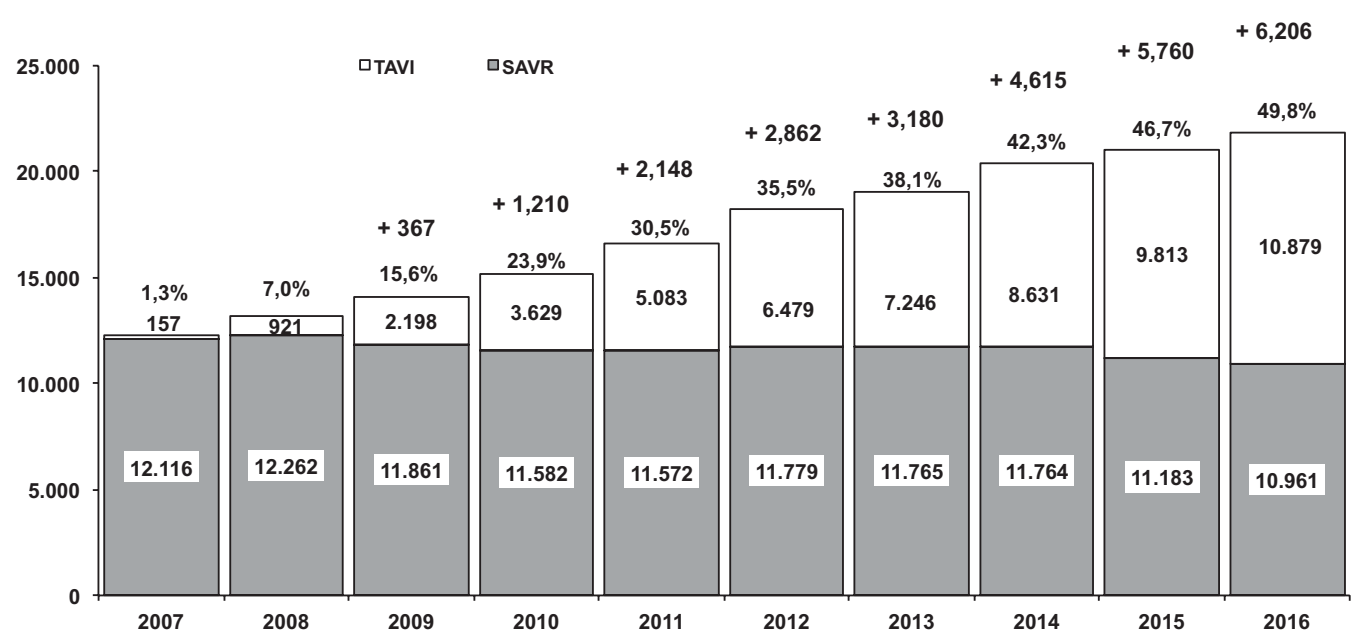

Fig. 5 Isolated aortic valve replacement and transcatheter aortic valve implantation (TAVI) The annual count of TAVI submitted to the voluntary registry of German Society for Thoracic and Cardiovascular Surgery does not represent all TAVI procedures performed in Germany in 2016. ${ }^{+}$Additional TAVI procedures calculated from the German legal quality assurance program, § 137 SGB V. 


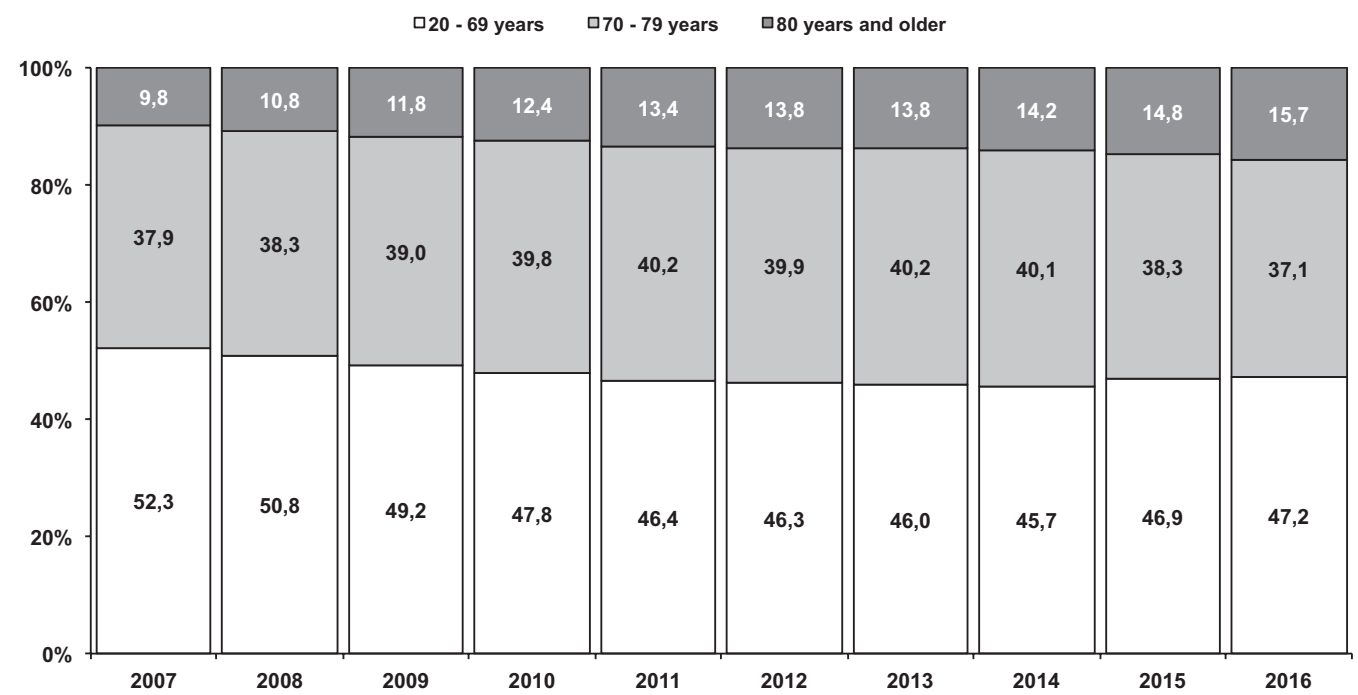

Fig. 6 Age distribution of cardiac procedures (2007-2016). Notes: Patients < 20 years and pacemaker/implantable cardioverter defibrillator procedures were excluded.

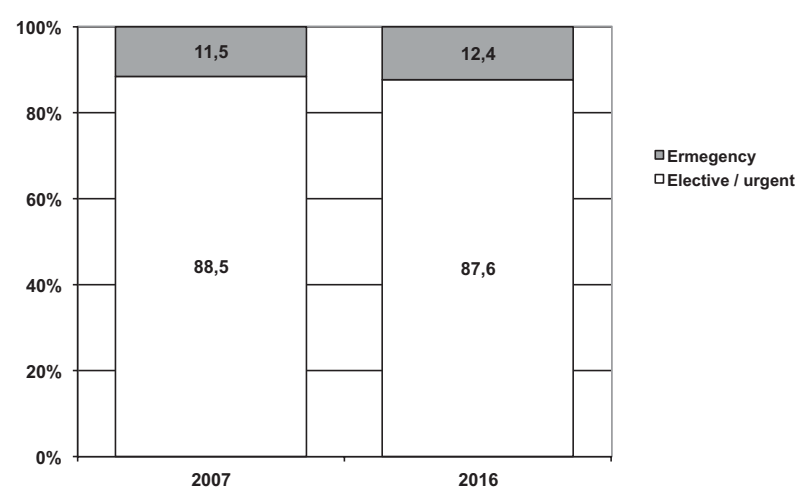

Fig. 7 Urgency categories (2007 vs. 2016) Emergency = acutely life-threatening cardiac/vascular disease.

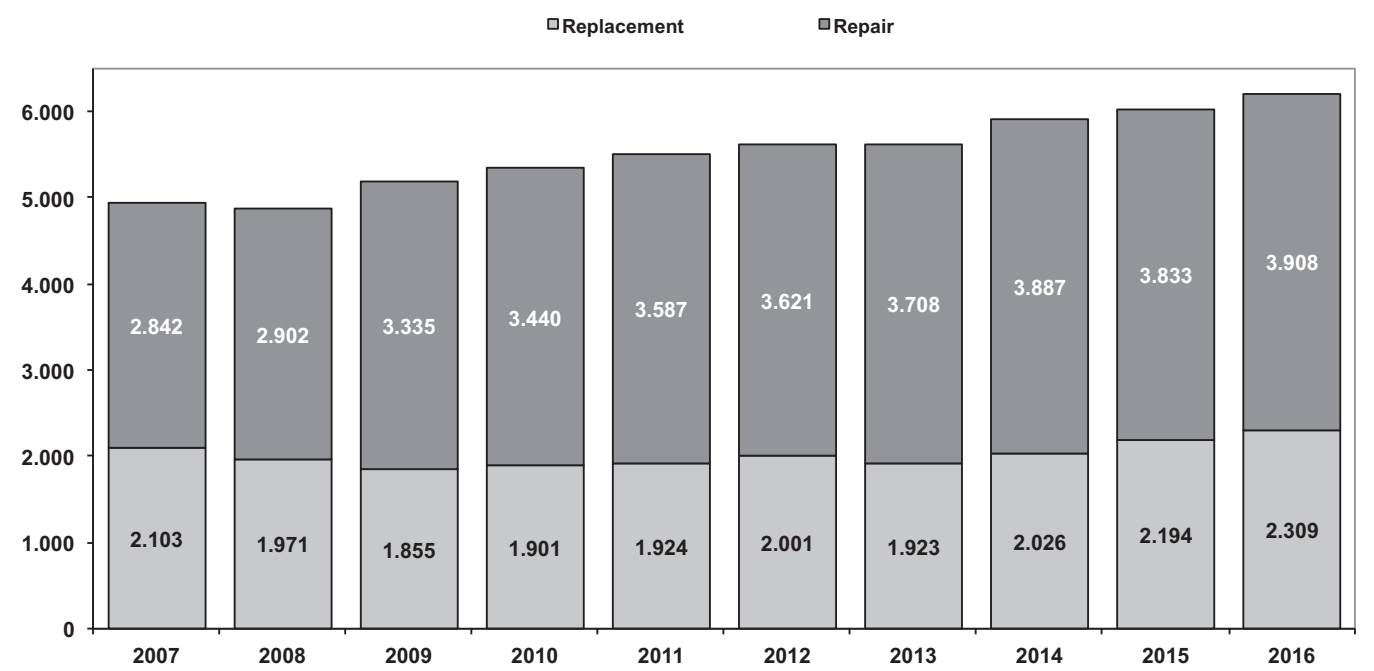

Fig. 8 Isolated mitral valve surgery (2007-2016). 


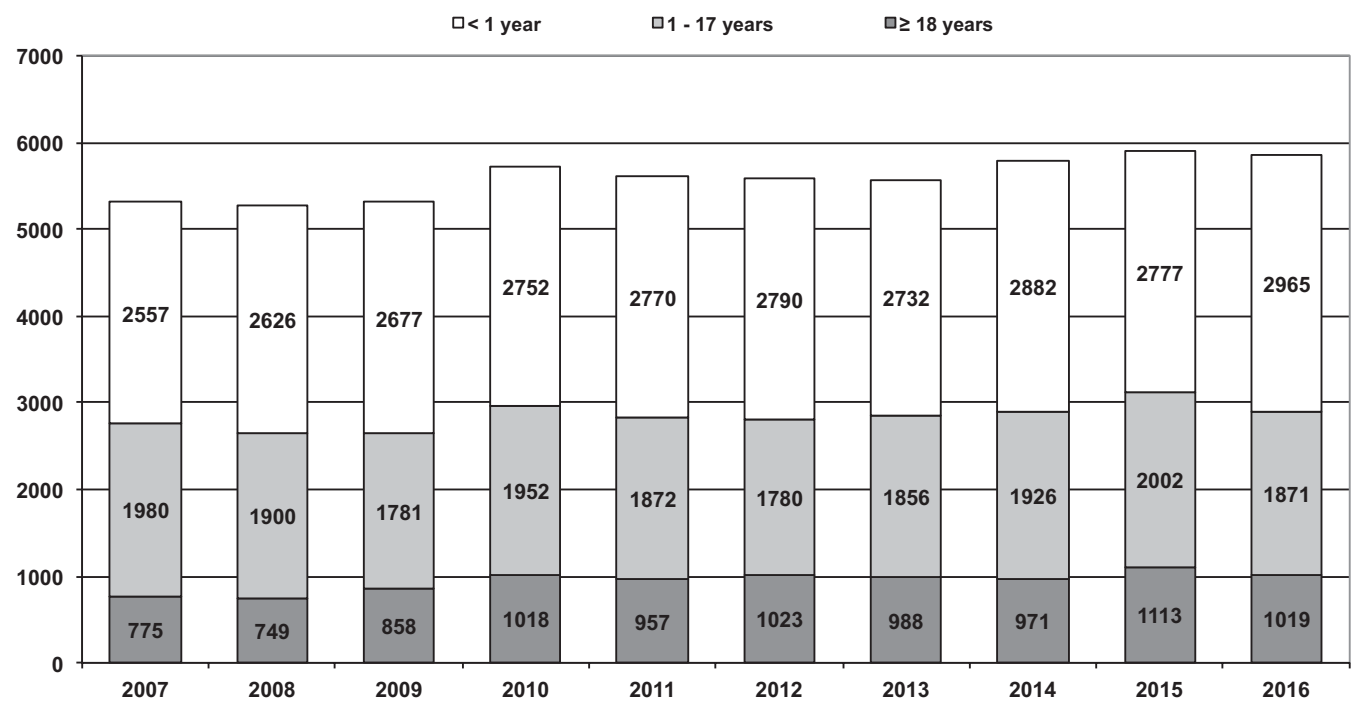

Fig. 9 Age distribution for CHD (2007-2016). Notes: Bias possible due to the fact that not all relevant procedures can be allocated exactly to CHD category in patients older than 18 years (e.g., aortic valve disease). CHD, congenital heart disease.

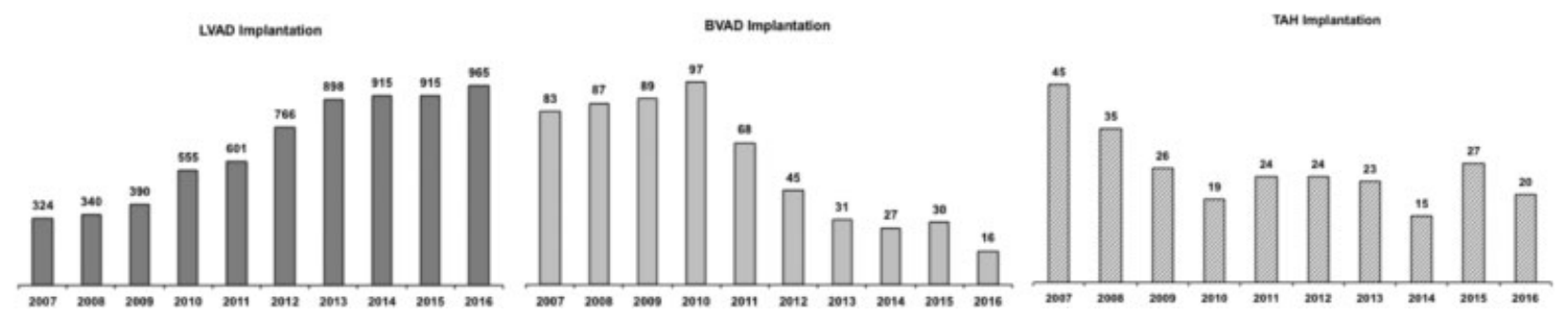

Fig. 10 Development of mechanical circulatory support (2007-2016). BVAD, biventricular assist device; LVAD, left ventricular assist device; TAH, total artificial heart.

\section{References}

1 Kalmar P, Irrgang E. Cardiac surgery in the Federal Republic of Germany during 1989. A report by the German Society for Thoracic and Cardiovascular Surgery. Thorac Cardiovasc Surg 1990;38(03):198-200

2 Kalmar P, Irrgang E. Cardiac surgery in the Federal Republic of Germany during 1990. A report by the German Society for Thoracic and Cardiovascular Surgery. Thorac Cardiovasc Surg 1991;39(03):167-169

3 Kalmar P, Irrgang E. Cardiac surgery in Germany during 1991. A report by the German Society for Thoracic and Cardiovascular Surgery. Thorac Cardiovasc Surg 1992;40(03):163-165

4 Kalmar P, Irrgang E. Cardiac surgery in Germany during 1992. A report by the German Society for Thoracic and Cardiovascular Surgery. Thorac Cardiovasc Surg 1993;41(03):202-204

5 Kalmar P, Irrgang E. Cardiac surgery in Germany during 1993. A report by the German Society for Thoracic and Cardiovascular Surgery. Thorac Cardiovasc Surg 1994;42(03):194-196

6 Kalmar P, Irrgang E. Cardiac surgery in Germany during 1994. A report by the German Society for Thoracic and Cardiovascular Surgery. Thorac Cardiovasc Surg 1995;43(03):181-183

7 Kalmár P, Irrgang E. Cardiac surgery in Germany during 1995. A report by the German Society for Thoracic and Cardiovascular Surgery. Thorac Cardiovasc Surg 1996;44(03):161-164

8 Kalmár P, Irrgang E. Cardiac surgery in Germany during 1996. A report by the German Society for Thoracic and Cardiovascular Surgery. Thorac Cardiovasc Surg 1997;45(03):134-137
9 Kalmár P, Irrgang E. Cardiac surgery in Germany during 1997. A report by the German Society for Thoracic and Cardiovascular Surgery. Thorac Cardiovasc Surg 1998;46(05):307-310

10 Kalmàr P, Irrgang E. Cardiac surgery in Germany during 1998. A report by the German Society for Thoracic and Cardiovascular Surgery. Thorac Cardiovasc Surg 1999;47(04):260-263

11 Kalmár P, Irrgang E. Cardiac surgery in Germany during 1999. Thorac Cardiovasc Surg 2000;48(04):XXVII-XXX

12 Kalmar P, Irrgang E. Cardiac surgery in Germany during 2000. Thorac Cardiovasc Surg 2001;49(05):XXXIII-XXXVIII

13 Kalmár P, Irrgang E. Cardiac surgery in Germany during 2001: a report by the German Society for Thoracic and Cardiovascular Surgery. Thorac Cardiovasc Surg 2002;50(06):30-35

14 Kalmár P, Irrgang E; German Society for Thoracic and Cardiovascular Surgery. Cardiac surgery in Germany during 2002: a report by German Society for Thoracic and Cardiovascular Surgery. Thorac Cardiovasc Surg 2003;51(05):25-29

15 Kalmár P, Irrgang E; German Society for Thoracic and Cardiovascular Surgery. Cardiac surgery in Germany during 2003: a report by the German Society for Thoracic and Cardiovascular Surgery. Thorac Cardiovasc Surg 2004;52(05):312-317

16 Gummert JF, Funkat A, Krian A. Cardiac surgery in Germany during 2004: a report on behalf of the German Society for Thoracic and Cardiovascular Surgery. Thorac Cardiovasc Surg 2005;53(06):391-399

17 Gummert JF, Funkat A, Beckmann A, Hekmat K, Ernst M, Krian A. Cardiac surgery in Germany during 2005: a report on behalf of the 
German Society for Thoracic and Cardiovascular Surgery. Thorac Cardiovasc Surg 2006;54(05):362-371

18 Gummert JF, Funkat A, Beckmann A, et al. Cardiac surgery in Germany during 2006: a report on behalf of the German Society for Thoracic and Cardiovascular Surgery. Thorac Cardiovasc Surg 2007;55(06):343-350

19 Gummert JF, Funkat A, Beckmann A, et al; German Society for Thoracic and Cardiovascular Surgery. Cardiac surgery in Germany during 2007: a report on behalf of the German Society for Thoracic and Cardiovascular Surgery. Thorac Cardiovasc Surg 2008;56(06):328-336

20 Gummert JF, Funkat A, Beckmann A, et al. Cardiac surgery in Germany during 2008. A report on behalf of the German Society for Thoracic and Cardiovascular Surgery. Thorac Cardiovasc Surg 2009;57(06):315-323

21 Gummert JF, Funkat A, Beckmann A, et al. Cardiac surgery in Germany during 2009. A report on behalf of the German Society for Thoracic and Cardiovascular Surgery. Thorac Cardiovasc Surg 2010;58(07):379-386

22 Gummert JF, Funkat AK, Beckmann A, et al. Cardiac surgery in Germany during 2010: a report on behalf of the German Society for Thoracic and Cardiovascular Surgery. Thorac Cardiovasc Surg 2011;59(05):259-267

23 Funkat AK, Beckmann A, Lewandowski J, et al. Cardiac surgery in Germany during 2011: a report on behalf of the German Society for Thoracic and Cardiovascular Surgery. Thorac Cardiovasc Surg 2012;60(06):371-382

24 Beckmann A, Funkat AK, Lewandowski J, et al. Cardiac surgery in Germany during 2012: a report on behalf of the German Society for Thoracic and Cardiovascular Surgery. Thorac Cardiovasc Surg 2014;62(01):5-17

25 Funkat A, Beckmann A, Lewandowski J, et al. Cardiac surgery in Germany during 2013: a report on behalf of the German Society for Thoracic and Cardiovascular Surgery. Thorac Cardiovasc Surg 2014;62(05):380-392

26 Beckmann A, Funkat AK, Lewandowski J, et al. Cardiac surgery in Germany during 2014. A report on behalf of the German Society for Thoracic and Cardiovascular Surgery. Thorac Cardiovasc Surg 2015;63(04):258-269

27 Beckmann A, Funkat AK, Lewandowski J, et al. German Heart Surgery Report 2015: the annual updated registry of the German Society for Thoracic and Cardiovascular Surgery. Thorac Cardiovasc Surg 2016;64(06):462-474

28 Vahanian A, Alfieri O, Andreotti F, et al; Joint Task Force on the Management of Valvular Heart Disease of the European Society of Cardiology (ESC); European Association for Cardio-Thoracic Surgery (EACTS). Guidelines on the management of valvular heart disease (version 2012). Eur Heart J 2012;33(19):2451-2496

29 Nishimura RA, Otto CM, Bonow RO, et al; ACC/AHA Task Force Members. 2014 AHA/ACC guideline for the management of patients with valvular heart disease: a report of the American College of Cardiology/American Heart Association Task Force on Practice Guidelines. Circulation 2014;129(23):e521-e643

30 Nishimura RA, Otto CM, Bonow RO, et al. 2017 AHA/ACC focused update of the 2014 AHA/ACC guideline for the management of patients with valvular heart disease: a report of the American College of Cardiology/American Heart Association Task Force on Clinical Practice Guidelines. Circulation 2017;135(25): e1159-e1195

31 Beckmann A, Hamm C, Figulla HR, et al; GARY Executive Board. The German Aortic Valve Registry (GARY): a nationwide registry for patients undergoing invasive therapy for severe aortic valve stenosis. Thorac Cardiovasc Surg 2012;60(05):319-325

32 Hamm CW, Möllmann H, Holzhey D, et al; GARY-Executive Board. The German Aortic Valve Registry (GARY): in-hospital outcome. Eur Heart J 2014;35(24):1588-1598

33 Mohr FW, Holzhey D, Möllmann H, et al; GARY Executive Board. The German Aortic Valve Registry: 1-year results from 13,680 patients with aortic valve disease. Eur J Cardiothorac Surg 2014; 46(05):808-816

34 Lauten A, Figulla HR, Möllmann H, et al; GARY Executive Board. TAVI for low-flow, low-gradient severe aortic stenosis with preserved or reduced ejection fraction: a subgroup analysis from the German Aortic Valve Registry (GARY). EuroIntervention 2014;10 (07):850-859

35 Walther T, Hamm C, Schuler G, et al; GARY Executive Board. Perioperative Results and Complications in 15,964 Transcatheter Aortic Valve Replacements: Prospective Data From the GARY Registry. J Am Coll Cardiol 2015;65(20):2173-2180

36 Holzhey D, Mohr FW, Walther T, et al. Current results of surgical aortic valve replacement: insights from the German Aortic Valve Registry. Ann Thorac Surg 2016;101(02):658-666

37 Lange R, Beckmann A, Neumann T, et al; GARY Executive Board. Quality of life after transcatheter aortic valve replacement: prospective data from GARY (German Aortic Valve Registry). JACC Cardiovasc Interv 2016;9(24):2541-2554

38 Bauer T, Möllmann H, Beckmann A, et al. Left ventricular function determines the survival benefit for women over men after transcatheter aortic valve implantation (TAVI). EuroIntervention 2017;13(04):467-474

39 Gammie JS, Zhao Y, Peterson ED, O'Brien SM, Rankin JS, Griffith BPJ. J. Maxwell Chamberlain Memorial Paper for adult cardiac surgery. Less-invasive mitral valve operations: trends and outcomes from the Society of Thoracic Surgeons Adult Cardiac Surgery Database. Ann Thorac Surg 2010;90(05):1401-1408, 1410.e1, discussion 1408-1410

40 Herbert MA, Prince SL, Williams JL, Magee MJ, Mack MJ. Are unaudited records from an outcomes registry database accurate? Ann Thorac Surg 2004;77(06):1960-1964, discussion 1964-1965 